\title{
Predicting storm-time thermospheric mass density variations at CHAMP and GRACE altitudes
}

\author{
R. Liu ${ }^{1,2}$, S.-Y. Ma ${ }^{1}$, and H. Lühr ${ }^{2}$ \\ ${ }^{1}$ Dept. of Space Physics, College of Electronic Information, Wuhan University, Wuhan 430079, China \\ ${ }^{2}$ Helmholtz Center Potsdam, GFZ German Research Center for Geosciences, Telegrafenberg, 14473 Potsdam, Germany
}

Received: 26 September 2010 - Revised: 18 February 2011 - Accepted: 22 February 2011 - Published: 1 March 2011

\begin{abstract}
Orbit-averaged mass density measurements derived from the satellites CHAMP and GRACE are used to investigate the storm-time prediction model developed by Liu et al. (2010) at different altitudes. This model uses as input only the solar wind merging electric field. From 2002 to 2005 in total 31 major geomagnetic storms with minimum Dst $<-100 \mathrm{nT}$ are selected for a statistical study. The results show that the model can successfully predict the storm-time mass density changes at both CHAMP and GRACE altitudes. The orbit-averaged density of CHAMP and GRACE show very similar distribution in shape, regardless of the orbital local time difference, but the amplitude of GRACE density is about $30 \%$ of that of CHAMP density. An optimal delay time of $4.5 \mathrm{~h}$ has been found for both CHAMP and GRACE densities. During the four years the scale factor $a$ between merging electric field and mass density for CHAMP altitude remains basically at the same level, $a=0.5$, while the $a$ for GRACE density shows a declining trend, and its value is only $30 \%$ of that for CHAMP density. The storms driven by corotating interaction regions (CIR) have in general larger $a$ values than the storms driven by coronal mass ejections (CMEs).
\end{abstract}

Keywords. Ionosphere (Ionosphere-atmosphere interactions) - Magnetospheric physics (Solar wind-magnetosphere interactions; Storms and substorms)

\section{Introduction}

The study of thermospheric mass density has been a topic of great importance since more and more spacecraft are launched into the height range of several hundred kilometers altitude. Understanding the behavior of thermospheric

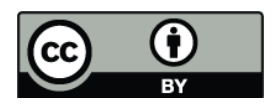

Correspondence to: R. Liu (liuruosi@whu.edu.cn) mass density is essential for the control of spacecraft flying in low-Earth orbit. The thermospheric mass density is a rather variable quantity which reacts to changing solar and geomagnetic conditions. During geomagnetic storms a larger amount of energy is deposited in the upper atmosphere at high latitudes in the form of Joule heating and particle precipitation. The local heating of neutral particles leads to upwelling in the source region. Energy and momentum are redistributed in the form of global meridional circulations and large-scale traveling atmospheric disturbances (TAD) (Bruinsma and Forbes, 2007). A series of complex physical processes lead to strong disturbances of the thermospheric mass density during magnetic storms (e.g. Liu and Lühr, 2005).

For decades studies have been dedicated to the subject of the thermosphere response to geomagnetic storms (e.g. Matuura, 1972; Romanoysky and Katyushina, 1974; Prölss, 1980; Crowley, 1991; Fuller-Rowell et al., 1994; Forbes et al., 1996; Burke et al., 2009). After the launch of the satellites CHAMP in 2000 and GRACE in 2002, new comprehensive and high-resolution datasets enable us to study the thermosphere on a long-term basis and from the global point of view. Plenty of studies made use of the density inferred from the measurements of accelerometer on board CHAMP and GRACE to analyze the response of the thermosphere to geomagnetic storms (e.g. Bruinsma et al., 2004; Liu and Lühr, 2005; Forbes et al., 2005; Sutton et al., 2005; Burke et al., 2007). In fact, not only the sole measurements from either CHAMP or GRACE have opened lots of possibilities, but the coexistence of CHAMP and GRACE since March 2002 also provides us an unprecedented opportunity to study the longterm density variation at $400 \mathrm{~km}$ and $500 \mathrm{~km}$ simultaneously. For example, Bruinsma et al. (2006) have used the density measurements from CHAMP and GRACE to investigate the global density response during the severe magnetic storm of 19 to 21 November 2003, and compared the results with the predictions of the NRLMSIS-00 model. 
More recently there are many studies focusing on the topic of investigating the response of thermospheric density, or the thermosphere itself, to some particular geomagnetic drivers. It is well-known that the predictions by empirical atmospheric models, such as the MSIS model series (Hedin, 1991; Picone et al., 2002), of density changes during geomagnetic storms is still not satisfying. Studying the density response to various geomagnetic drivers may be helpful for the improvement of the current density models as well as for establishing new models. One of the most commonly considered driver is Joule heating. Sutton et al. (2009) have studied the CHAMP density correlation with high-latitude heating sources by investigating the response time of the thermosphere to a derived Joule heating index for a series of three geomagnetic storms during the period of 20 to 29 July 2004. Zhou et al. (2009) also have studied the thermospheric density response to Joule heating in terms of the total global Joule heating power, $\Sigma Q_{j}$, and the high resolution ring current index, Sym-H. They have corrected the storm-time predictions of the NRLMSISE-00 model with $\Sigma Q_{j}$ and Sym-H, based on the results of a statistical study using CHAMP mass density data from 19 great magnetic storms during 2001 to 2004. Although Joule heating is most directly related to the energy input during geomagnetic storms, the calculation of Joule heating is complicated and not unambiguous. It requires the computation of Pedersen conductivity and convection electric field, both of which are calculated from empirical models. This is on one hand inconvenient, on the other hand not precise, due to the uncertainties of the models. So the question is whether there is a physical quantity which can be obtained from observational data and is easy to be calculated.

Liu et al. (2010) have found that the storm-time density changes at $400 \mathrm{~km}$ correlate well with the merging electric field $\boldsymbol{E}_{\mathrm{m}}=v_{\mathrm{SW}} B_{\mathrm{T}} \sin ^{2}(\theta / 2)$, where $v_{\mathrm{SW}}$ is the solar wind speed, $B_{\mathrm{T}}=\sqrt{{B_{\mathrm{y}}}^{2}+B_{\mathrm{z}}{ }^{2}}$ is the transverse component of IMF in Geocentric-Solar-Magnetospheric (GSM) coordinates, $\theta$ is the IMF clock angle defined by $\tan (\theta)=\left|B_{\mathrm{y}}\right| / B_{\mathrm{Z}}(0 \leq \theta \leq$ $\pi$ ). Liu et al. (2010) utilized the density measurements of CHAMP to study the response of the total mass density at $400 \mathrm{~km}$ to the merging electric field $\boldsymbol{E}_{\mathrm{m}}$ during magnetic storms. Based on an integrated merging electric field $\bar{E}_{\mathrm{m}}$ and considering a delayed response of the density a linear prediction model particularly for storm time is developed. Since $\boldsymbol{E}_{\mathrm{m}}$ is derived directly from satellite observations of solar wind and IMF data, it is not dependent on any assumptions. Therefore it is superior to the estimated Joule heating for predicting storm-time mass density variations. The main empirical conclusions yielded by Liu et al. (2010) are:

1. The thermospheric density at $400 \mathrm{~km}$ is linearly correlated with the time-delayed and integrated merging electric field $\bar{E}_{\mathrm{m}}$ throughout all storm phases.
2. A truncation of $\bar{E}_{\mathrm{m}}$ reflecting the saturation effect of the transpolar potential does not help to track the density variations well, especially during the super storms. Only when the truncation is removed, $\bar{E}_{\mathrm{m}}$ maintains a simple linear relation with density.

3. The delay time of the density with respect to $\bar{E}_{\mathrm{m}}$ shows dependences on geomagnetic latitude and local time. A constant density delay time of $3 \mathrm{~h}$ is used at low latitudes, and a local time dependent delay time between 0 to $4.5 \mathrm{~h}$ is found at mid latitudes for day and night side, respectively.

4. The linear prediction model can be expressed by the equation

$$
\rho=a \bar{E}_{\mathrm{m}}+\rho_{\mathrm{amb}}
$$

the proportionality factor $a$ is independent of local time. For CHAMP altitude $a=0.5$ can be used for all the storms during 2002 to 2005 . The ambient density, $\rho_{\mathrm{amb}}$, is determined from the quiet day before the storm. This linear relation can reproduce the storm-time density changes sufficiently well.

5. The orbit-averaged density can be better reproduced by the prediction model than the density variations in the different latitudinal zones.

The presented work is an extension of the study by Liu et al. (2010). The motivation to carry out further analysis is (1) in Liu et al. (2010) the performance of CHAMP orbitaveraged density $\rho_{\text {avg }}$ has not been fully discussed, (2) Liu et al. (2010) only took the CHAMP density measurements into account, hence the derived model can only be used for the densities around $400 \mathrm{~km}$ altitude. The validity of the model at other altitudes (e.g. the altitude of GRACE) needs to be tested. In the present paper the same method for deducing the prediction model presented in Liu et al. (2010) is used to further analyze the dependence of $\rho_{\text {avg }}$ from CHAMP as well as from GRACE on $\bar{E}_{\mathrm{m}}$ during geomagnetic storms.

In the next section we will introduce the dataset used in this study. The properties and predictions of the model at CHAMP and GRACE altitudes are shown in Sect. 3. After that the results are discussed and summarized in Sects. 4 and 5 .

\section{Dataset}

In this paper the total mass density deduced from measurements of the accelerometers aboard the two satellites CHAllenging Minisatellite Payload (CHAMP) and Gravity Recovery And Climate Experiment (GRACE) are used to investigate the thermospheric density response to $\boldsymbol{E}_{\mathrm{m}}$ during geomagnetic storms. The advantage of considering both 
CHAMP and GRACE measurements is that we can monitor the density response at two different altitudes simultaneously and make comparisons afterwards. CHAMP was launched on 15 July 2000 into a near-polar orbit with an inclination of $87.25^{\circ}$. The initial altitude of the CHAMP orbit is $425 \mathrm{~km}$. The orbital period of CHAMP is about $93 \mathrm{~min}$. Within 131 days the orbit covers all local times. GRACE was launched on 17 March 2002. The GRACE mission consists of two identical spacecraft, GRACE-A and GRACE-B, flying in tandem at a distance of about $220 \mathrm{~km}$ in a near-circular orbit with an inclination of $89.5^{\circ}$ and at an altitude around $500 \mathrm{~km}$. In this study only the measurements of GRACE-A are used, in the following we refer to the GRACE-A measurements as GRACE for convenience's sake. GRACE has an orbital period of $95 \mathrm{~min}$. The orbit of GRACE covers all local times within 160 days.

Due to their long-term measurements with high timeresolution, and their orbital coverage of all local times and latitudes, both CHAMP and GRACE are very suitable for long-term monitoring and for improving the global profile of the thermosphere. Both CHAMP and GRACE are equipped with Space Triaxial Accelerometer for Research (STAR) (SuperSTAR in case of GRACE). The acceleration measured by STAR reflects the non-gravitational acceleration acting on the satellite due to air drag, solar radiation pressure or attitude maneuvers. The thermospheric mass density is deduced from the acceleration due to air drag. For this task the STAR measurements are preprocessed by removing acceleration components caused by solar radiation pressure and attitude maneuvers. For our study we make use of the preprocessed accelerometer data. The density is determined by projecting the total acceleration onto the $\mathrm{x}$-axis of the instrument (along-track component). The calculation algorithms will not be repeated here, since it is already described in Liu et al. (2010). For a more comprehensive and detailed description of accelerometer data processing the reader is referred to Doornbos et al. (2010). The time resolution of the determined density data is $10 \mathrm{~s}$.

The calculation of the merging electric field requires the solar wind and IMF data, which are taken from the Advanced Composition Explorer (ACE) spacecraft. ACE is an explorer mission that was launched on 25 August 1997 and positioned near the L1 Lagrangian point about 1.5 million $\mathrm{km}$ from Earth and 148.5 million $\mathrm{km}$ from the Sun. The data we used are solar wind data shifted in time to the front side magnetopause, which is assumed to be located at a distance of $10 R_{\mathrm{E}}$ at the sub-solar point. The data resolution is $1 \mathrm{~min}$. Normally it takes 20 to $60 \mathrm{~min}$ for the solar wind features observed by ACE to arrive at the Earth's magnetopause. The propagation time is calculated using the so-called phase front propagation technique (Weimer et al., 2003).

For this study the four years from 2002 to 2005 are chosen as the time interval of interest because CHAMP and GRACE measurements are well overlapped during this period since March 2002. A large number of major magnetic
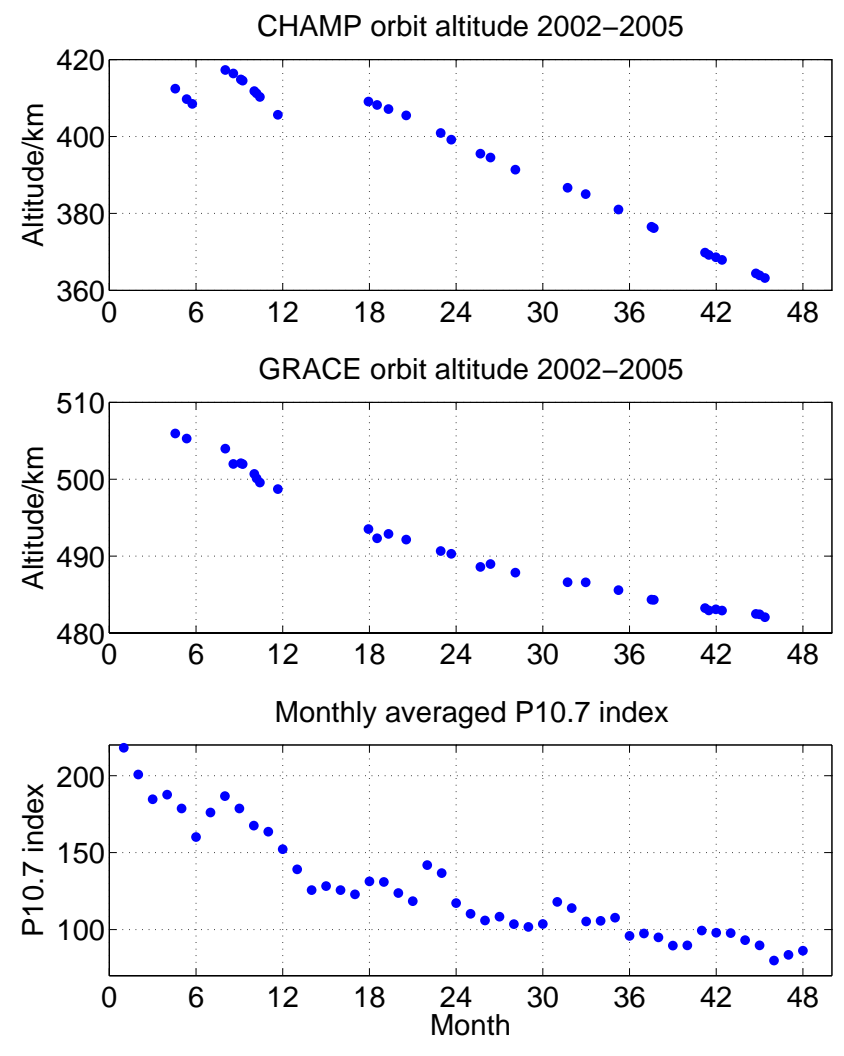

Fig. 1. The altitude (km) decays of CHAMP (top) and GRACE (middle) from April 2002 to September 2005. The x-axis represents the time starting from January 2002. Each dot represents the altitude averaged over one storm. In the bottom frame the evolution of the solar flux P10.7 is shown.

storms occurred after the solar maximum in 2001, whereas very few storms occur in the subsequent years. Altogether 31 major geomagnetic storms are selected with a minimum Dst $<-100 \mathrm{nT}$. These four years are in the declining phase of the solar cycle 23; the solar flux index P10.7 (see Eq. 2 for explanation) is decaying from 179 in 2002 to 92 in 2005 (see Fig. 1 bottom frame).

During 2002 to 2005 the altitudes of CHAMP and GRACE are both decreasing due to air drag. Figure 1 shows the respective orbital altitudes of CHAMP and GRACE for the 31 storms. From April 2002 to September 2005 CHAMP descended $54 \mathrm{~km}$ while GRACE descended only $24 \mathrm{~km}$. The altitude of CHAMP was raised two times in June and December 2002. From then on, the orbit decayed almost linearly to $363 \mathrm{~km}$ in September 2005 at a rate of $1.6 \mathrm{~km} / \mathrm{month}$. The altitude of GRACE shows a continuous and steady declination since no orbit change manoeuvre was performed. GRACE declined from $506 \mathrm{~km}$ in April 2002 to $482 \mathrm{~km}$ in September 2005. The rate of decay is getting smaller every year, from $1 \mathrm{~km} /$ month in 2002 to $0.25 \mathrm{~km} /$ month in 2005 . Compared to CHAMP the decline of GRACE is much slower. 

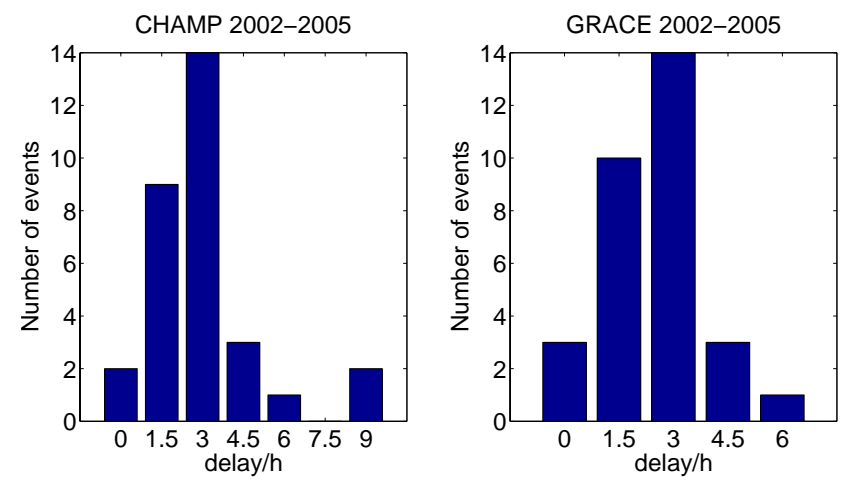

Fig. 2. The delayed orbit-averaged mass density response with respect to $\bar{E}_{\mathrm{m}}$ for CHAMP (left) and GRACE (right). The y-axis indicates the number of storm events per bin.

\section{Results}

In this section the density readings averaged over an orbit, $\rho_{\text {avg }}$, from CHAMP and GRACE are used to study the stormtime response to the preprocessed merging electric field. The density response is investigated in terms of delay time with respect to $\bar{E}_{\mathrm{m}}$ and the scaling factor $a$. The same dataprocessing method as described in Liu et al. (2010) is used. Here we would like to repeat the important parts only briefly: the density measurements at mid and low latitudes (between $\pm 60^{\circ}$ MLat) are averaged for each CHAMP/GRACE orbit; high latitude regions are excluded because the density here responds to heating and composition change during magnetic storms (e.g. Lei et al., 2010a). $\boldsymbol{E}_{\mathrm{m}}$ is integrated over a period of about $3 \mathrm{~h}$. After this preconditioning $\boldsymbol{E}_{\mathrm{m}}$ is termed $\bar{E}_{\mathrm{m}}$, which shows better correlation with $\rho_{\text {avg }}$.

Figure 2 shows the delay times determined individually for each storm by cross-correlation of the integrated $\bar{E}_{\mathrm{m}}$ with the observed density variations from both CHAMP (left) and GRACE (right). For the calculation of the correlation, the $\bar{E}_{\mathrm{m}}$ is delayed by $-1.5 \mathrm{~h}$ to $10.5 \mathrm{~h}$, in steps of $1.5 \mathrm{~h}$ (the orbit period of CHAMP/GRACE). The y-axis indicates the number of storm events per bin. The CHAMP delay times vary between 0 to $9 \mathrm{~h}$, i.e. up to 6 orbital periods. No negative value of time delay is observed, this means the density always reacts behind, instead of before, the changes of $\bar{E}_{\mathrm{m}}$, as expected. Out of the 31 storm events, 14 of them have a delay time of $3 \mathrm{~h}$, more than $70 \%$ of the events have a delay of $1.5 \mathrm{~h}$ or $3 \mathrm{~h}$. The distribution of delay times for GRACE shows a very similar pattern as that for CHAMP with only minor differences. The mean delay time for CHAMP and GRACE density is $3 \mathrm{~h}$ and $2.5 \mathrm{~h}$, respectively. So the density at $500 \mathrm{~km}$ altitude seems to react to the changes of geomagnetic conditions slightly faster than the density at $400 \mathrm{~km}$. We are, however, not convinced about that the significance of that difference.

The $a$ values for CHAMP and GRACE are shown in Fig. 3 . The scaling factor $a$ is determined by linear regression anal-
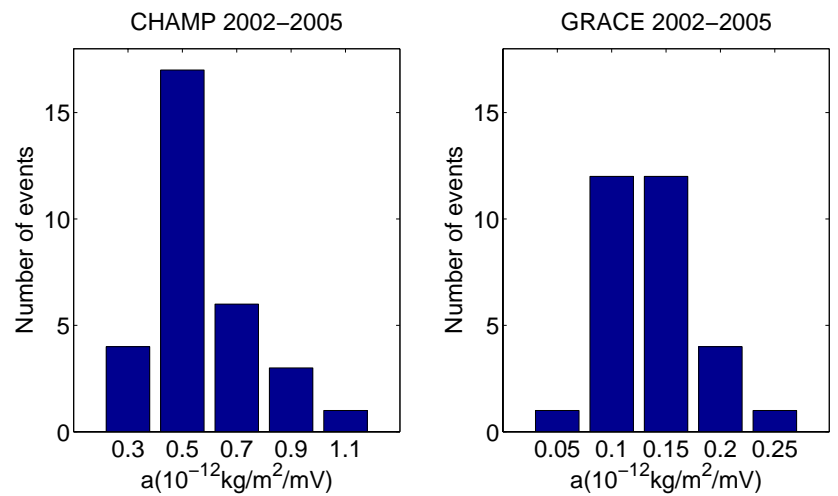

Fig. 3. The distribution of scaling factor $a$ for CHAMP (left) and GRACE (right). The y-axis indicates the number of storm events per bin.

ysis for each storm individually. It signifies the efficiency of $\bar{E}_{\mathrm{m}}$ in changing the density. The values of $a$ for CHAMP are sorted into 5 ranges of constant width 0.2 with respective median values from 0.3 to 1.1 as marked along the $\mathrm{x}$-axis. Similarly to the delay time, $a$ also exhibits a centralized distribution. Nearly $50 \%$ of the $a$ values are located in the range 0.5 , then the number of events is decreasing towards both sides of larger and smaller values. Events with $a \geq 1.0 \mathrm{can}$ hardly be found. The $a$ values for GRACE are also divided into five ranges of constant width 0.05 with median values from 0.05 to 0.25 . Over $70 \%$ of the events are located in the ranges 0.1 and 0.15 .

Figure 3 provides a first impression of the $a$ value distributions, whereas Fig. 4 shows the temporal evolution of $a$ for CHAMP and GRACE. Each dot represents one storm. The dots are coded by three different colors: blue indicates storms driven by interplanetary coronal mass ejections (ICMEs, the interplanetary manifestations of coronal mass ejections (CMEs) at the sun), green indicates storms generated by corotating interaction regions (CIR), and red indicates three super storms: the storm of 29-31 October 2003 with $\mathrm{Dst}_{\min }=-383 \mathrm{nT}$, the storm of 20-22 November 2003 with Dst $_{\min }=-422 \mathrm{nT}$ and the storm of 7-11 November 2004 with Dst $_{\min }=-373 \mathrm{nT}$. The three super storms are ICMEsdriven storms. It can be seen from the top panel that $a$ for CHAMP remains in general at the same level during the four years, no evidence for long-term trend is observed, except for the relatively higher values in 2005 . The annual mean values of $a$ are 0.55 in 2002, 0.46 in 2003, 0.51 in 2004, and 0.68 in 2005 . Therefore, in $2005 a$ is at least $30 \%$ higher than during the previous three years. Besides, the years 2003 and 2004 seem to have smaller variation scales of $a$ than the other two years: in 2003 and $2004 a$ is basically between 0.3 to 0.7 , with corresponding standard deviations of $8 \%$ and $11 \%$, while it varies in 2002 between 0.2 to 0.9 , in 2005 between 0.4 to 1.1 , with corresponding standard deviations of $16 \%$ and $17 \%$, respectively. If one looks into the CIR-driven 
storms marked by green color, one finds that their $a$ values are overall larger than those for the ICME-driven storms marked by blue color. This trend is more evident in 2002 and 2005. All three super storms have an $a$ value approximately fitting the average value 0.5 . Another thing worth to notice is that the storm in January 2005 has an extremely high $a$ value above 1.0. This ICME-driven storm occurred during 21 to 22 January 2005 with a minimum Dst of $-105 \mathrm{nT}$. Although Dst reached only a fairly moderate value, the solar wind dynamic pressure went up to an extraordinarily high value of $86 \mathrm{nPa}$, whilst the normal value of solar wind pressure is below $5 \mathrm{nPa}$. In addition, the high level of pressure lasts for a long time, the full width at half maximum (FWHM) of the peak is $70 \mathrm{~min}$. Possible explanations for the unusually high density enhancement during this storm will be discussed in the Sect. 4.4.

The temporal evolution of $a$ for GRACE seems to be different from that of CHAMP data. The $a$ values decline from year to year, as is shown in the bottom panel of Fig. 4. The mean value of $a$ is 0.19 in 2002, 0.15 in 2003, 0.12 in 2004, and 0.11 in 2005. The standard deviation of $a$ maintains the same level around 3\% to 5\% during the four years. Similarly, the CIR-driven storms have in general higher $a$ values than the ICME-driven storms. Yet, the storm on 21 January 2005 shows a high $a$ value well above the normal values, but it is no longer the largest value owing to the long-term decline. Except for the January storm, the values in 2005 are well-fitted by the long-term trend, the overall anomaly of $a$ in 2005 seen in the CHAMP result does not exist at GRACE altitude.

Figure 5 presents for four examples of comparisons between predictions and CHAMP density measurements, using a constant delay of $3 \mathrm{~h}$ and $a=0.5$. From each year one example is selected. The top left panel is for the storm in 3 to 5 September 2002, the top right panel for the storm 10 to 12 July 2003, the bottom left panel for the super storm 7 to 9 November 2004, and the bottom right for the storm 30 to 31 May 2005. Among the four storms, the 2002 and 2003 storms are CIR-driven storms, and the 2004 and 2005 storms are ICME-driven storms. The blue and red curves represent the CHAMP observations and model predictions, respectively. The good correlation between the observations and the predictions suggests that the use of $3 \mathrm{~h}$ delay time and $a=0.5$ in our model can provide good predictions for the storm-time density at CHAMP altitude during the four years, even though the determined $a$ value fluctuates around the average line. The predictions follow the changes of the density in all storm phases in both ICME-driven and CIR driven storms rather well. For the super storm in 7 to 9 November 2004, the prediction shows a lagged effect compared to the density observation. This implies that $3 \mathrm{~h}$ delay is too long for this super storm, in which the density reacts almost immediately to the geomagnetic forcing. But for the rest of the storms $3 \mathrm{~h}$ is a very reasonable delay time.
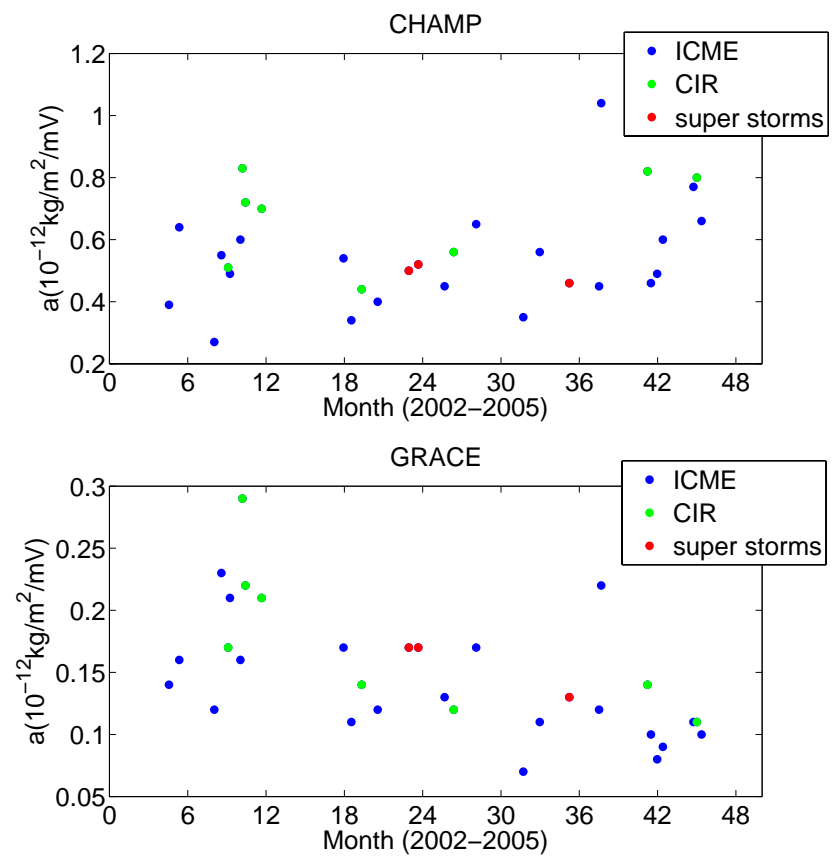

Fig. 4. Temporal evolution of the scaling factor $a$ derived individually for each storm by linear regression between CHAMP (top) and GRACE (bottom) density data and $E_{\mathrm{m}}$ during 2002 to 2005. Storm types are identified by color coding: blue for ICME-driven storms, green for CIR driven storms, red for the three super storms.

The predictions of GRACE density measurements for the same four storms are shown in Fig. 6. For GRACE data the constant delay time of $3 \mathrm{~h}$ is still suitable, but there is no constant value for $a$ due to the long-term tendency. We use the formula $a=0.19-0.0021 t$, which is derived from a linear fitting to the values shown in the bottom frame of Fig. 4, where the $a$ values for each individual storm is plotted. The time $t$ is in months from beginning of 2002. The applied $a$ values are listed in the top left corner of each panel. If we compare the density measurements of both satellites, the GRACE densities show very similar variations as the CHAMP densities in shape, even though in most storm events the two satellites orbit around the Earth at different local time sectors. But the amplitudes of GRACE densities are only $20 \%$ to $30 \%$ of the CHAMP densities. Taking the super storm in November 2004 as an example, the stormtime density along the CHAMP orbit reaches to a peak above $15 \times 10^{-12} \mathrm{~kg} \mathrm{~m}^{-3}$, while the density along the GRACE orbit reaches only above $3.5 \times 10^{-12} \mathrm{~kg} \mathrm{~m}^{-3}$. Exceptionally, in the 2005 storm, the GRACE peak density is only $13 \%$ of the highest density at CHAMP, which is consistent with the $a$ anomaly in 2005 observed in the top panel of Fig. 4. In Fig. 6 $a$ drops from 0.17 in 2002 to 0.09 in 2005 . The predictions can successfully reproduce the satellite observations if properly scaled.

Figure 7 gives the values of correlation coefficient $R$ between the prediction and the measurement for all the storms in the same format as Fig. 4. The result for CHAMP (top) is 

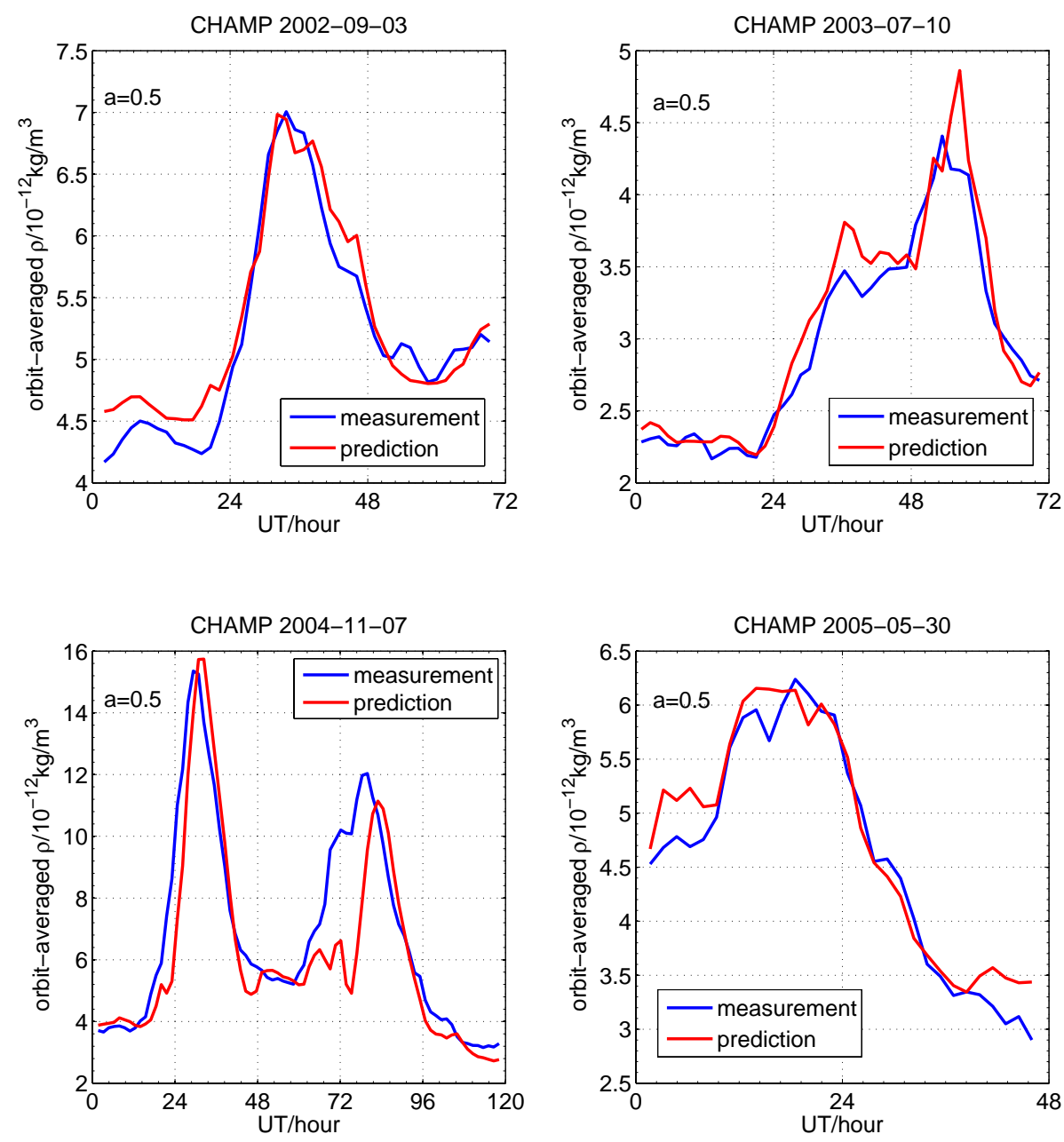

Fig. 5. Comparison between CHAMP orbit-averaged density measurements and predictions of our model using a density delay time of $3 \mathrm{~h}$ and $a=0.5$ for four storms during 2002 to 2005. 3 to 5 September 2002, 10 to 12 July 2003, 7 to 9 November 2004, and 30 to 31 May 2005 . The 2002 and 2003 storms are CIR driven storms, and the 2004 and 2005 storms are ICME-driven storms. The blue and red curves represent the CHAMP observations and model predictions, respectively.

quite similar as that of GRACE (bottom), while the predictions for GRACE show a slightly better correlation with the measurements. The $R$ for CHAMP has a mean value of 0.88 , with $80 \%$ of the values above 0.8 and $40 \%$ above 0.9 ; the mean value of $R$ for GRACE is 0.89 , with also $80 \%$ of the $R$ values above 0.8 , but $58 \%$ values above 0.9 . Figure 7 implies overall good prediction capability of the developed model at both CHAMP and GRACE altitudes. For CHAMP, except for the storm in April 2002, the smaller values, $R<0.8$, appear mainly within 2005, due to the larger deviation of $a$ from the baseline, $a=0.5$, in this year, as is evident from Fig. 4. The reduction of $R$ values in 2005 is not so evident for GRACE.

\section{Discussion}

We have performed a statistical analysis of the comparison between mass density variations during magnetic storms and predictions of a model developed by Liu et al. (2010). The 31 major storms occurring during 2002 to 2005 were considered. The results obtained by Liu et al. (2010) from CHAMP data could be confirmed, but by adding density measurements from the higher flying GRACE new issues arose. More general considerations are now required for providing a prediction model that is valid at both altitudes.

\subsection{What controls the scale factor $a$ ?}

In the previous study Liu et al. (2010) reported the stormtime density enhancement that can be described as an additive effect on top of the quiet-time background mass density. The enhancement is directly proportional to the preprocessed merging electric field $\bar{E}_{\mathrm{m}}$. By using CHAMP mass density measurements they found that a fixed scale factor, $a=0.5$, between density and $\bar{E}_{\mathrm{m}}$ yields suitable predictions for all magnetic storms during the years 2002 to 2005 . Here we 

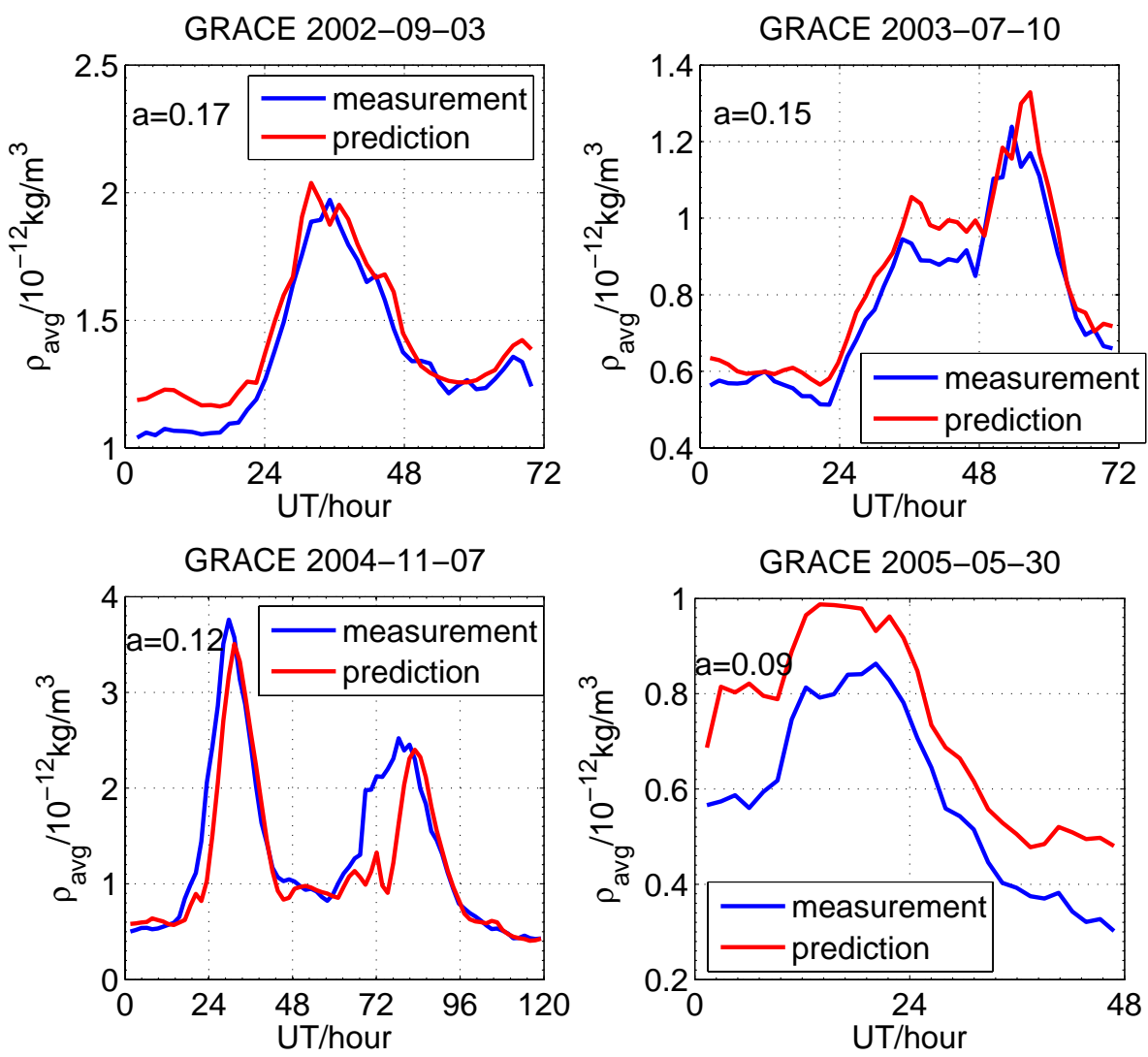

Fig. 6. The same format as Fig. 5, but for the GRACE density data. Different $a$ values are used for different storms. The $a$ value is shown in the top left corner of each panel.

have applied the same procedure to GRACE data from about $100 \mathrm{~km}$ higher. Our prediction model is equally efficient in predicting the density variations for those observations. Interestingly, the factor $a$ at GRACE altitude is on average only $30 \%$ of that found for CHAMP and the difference even increases with time. It appears understandable that $a$ is larger at lower altitude, but strangely the $a$ value for GRACE observations becomes smaller when the altitude gets lower over the years. For reconciling this apparent contradiction we may have a look at the evolution of the solar flux index P10.7 (see Fig. 1). P10.7 is calculated as:

$\mathrm{P} 10.7=\frac{1}{2}\left(\mathrm{~F} 10.7+\overline{\mathrm{F} 10.7_{81 \text { days }}}\right)$

(Richards et al., 1994). Compared to F10.7 it is a more appropriate indicator for the ionospheric and thermospheric response to solar EUV radiation (Liu et al., 2006; Rentz and Lühr, 2008). Obviously the scale factor $a$ is dependent both on altitude and on solar flux. For GRACE the decline in P10.7 overrides the effect of the orbit decay. Interestingly, in the case of CHAMP the decrease in solar flux and altitude compensate each other during the years 2002 to 2004. In the last year 2005 the orbit decay seems to be faster than the reduction in $\mathrm{P} 10.7$, resulting in a gradual increase of the factor $a$.

An immediate explanation could be that the value of $a$ is proportional to the background density. It was already shown by Liu et al. (2010) that this is not the case for CHAMP data. Our preferred suggestion is that $a$ varies with pressure level at least over the height range 350 to $500 \mathrm{~km}$ studied here. In order to test that proposition we calculated the ratios between the $a$ values of CHAMP and GRACE for each storm event. Figure 8 shows these ratios plotted versus the height difference between the spacecraft. As can be seen, all points group smoothly around a fitting curve of degree 4 , reaching 1 at the position of CHAMP. Unfortunately, the range of height difference covered is not too large, but the expected tendency is well recognizable.

For a more quantitative assessment of the ratio we have checked the height differences at certain ratios. The fitting curve takes the value $1 / e=0.37$ at an altitude difference of $83 \mathrm{~km}$. The ratio $1 / e^{2}=0.14$ is attained at $120 \mathrm{~km}$ difference. If we recall that small height differences occurred during 2002 when the sun was very active and large differences in 2005, a time of much reduced solar flux, the thermospheric scale height is also expected to have changed significantly during the considered years 2002 to 2005 . When assuming 

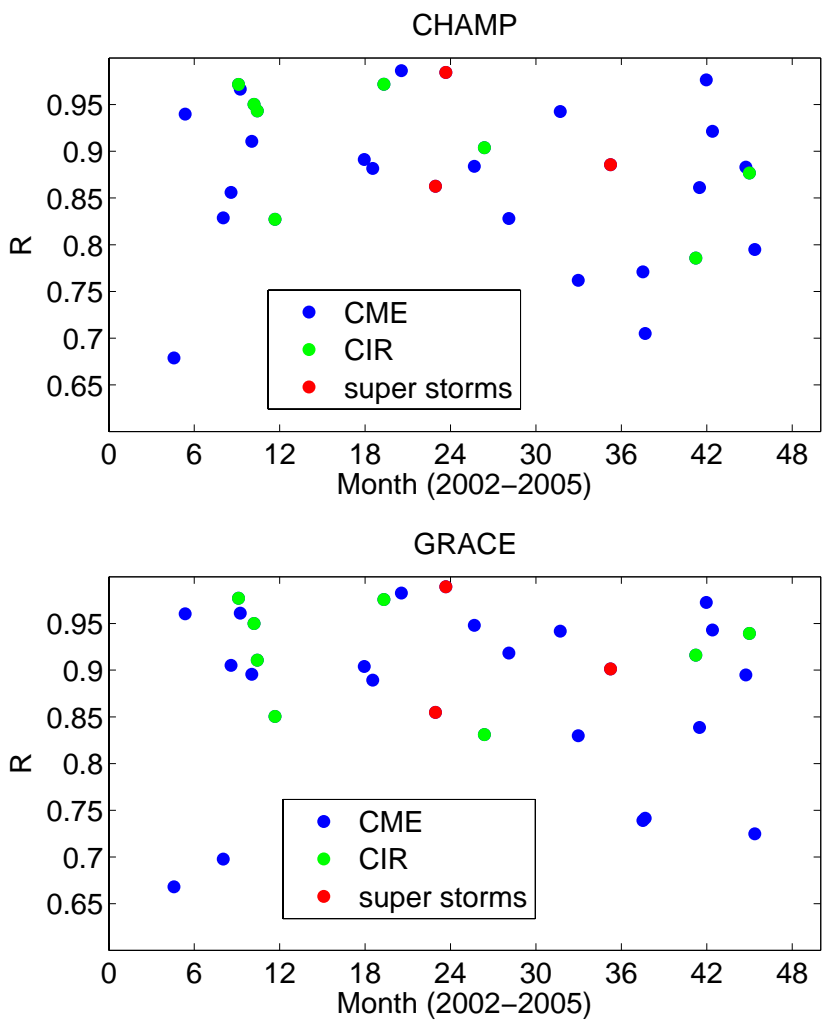

Fig. 7. The correlation coefficient $R$ between the prediction and the measurement for all the storms, in the same format as Fig. 4.

a direct dependence of the factor $a$ on pressure level, we obtain a scale height of $83 \mathrm{~km}$ for the beginning of 2002 (P10.7 > 200) and $60 \mathrm{~km}$ for the end of 2005 (P10.7 is around 80). These numbers are reasonably close to the expected pressure level scale heights under quoted conditions. For a verification of our suggestion physics-based numerical methods should be used.

Müller et al. (2009) have investigated the mass density enhancement due to magnetic activity. Using CHAMP data they correlated density variations with the magnetic activity index, $a_{\mathrm{m}}$. They also reported a simple linear relation between the two quantities. Judged from this study we can say, it was just the fortunate coincidence of CHAMP orbit decay and solar flux decline that they revealed a constant scaling factor for all the years. If they had taken GRACE data for their study, a time-dependent factor between $a_{\mathrm{m}}$ and density had emerged. This example shows the value of multispacecraft studies for characterizing dependences.

\subsection{The delayed response of the density at CHAMP and GRACE altitudes}

In Liu et al. (2010) a constant delay of $3 \mathrm{~h}$ is used for the density averaged over the low latitude area of $\pm 30^{\circ}$. In this study the $3 \mathrm{~h}$ delay has also proved to be an optimal delay time for the orbit-averaged density along both CHAMP and

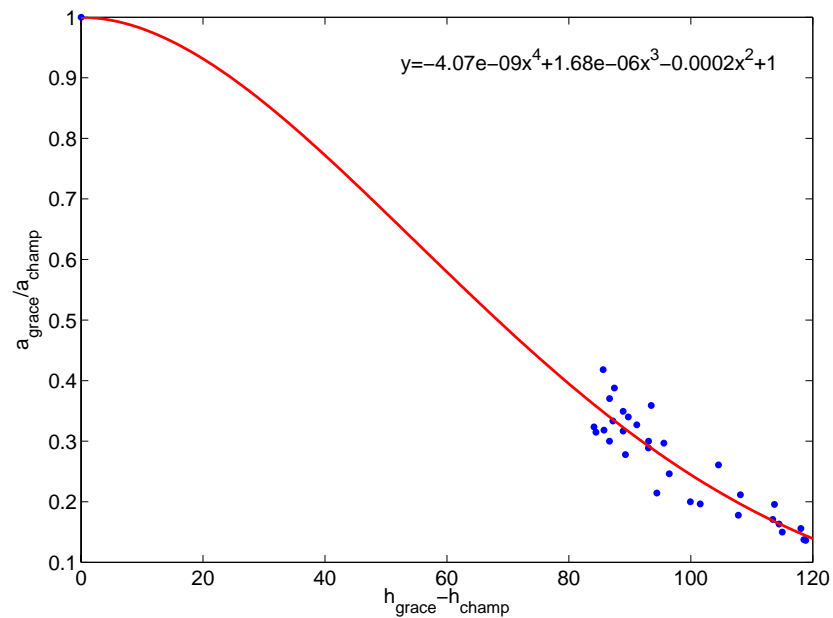

Fig. 8. The dependence of the ratio of the scale factor $a$ from CHAMP and GRACE on the height difference between the two spacecraft.

GRACE orbits, as shown in Fig. 2. One thing to note is, the $3 \mathrm{~h}$ delay in this paper is the delay between $\bar{E}_{\mathrm{m}}$ and the thermospheric density. The calculation of $\bar{E}_{\mathrm{m}}$ using Eq. (8) of Liu et al. (2010) produces already around $1.5 \mathrm{~h}$ delay of $\bar{E}_{\mathrm{m}}$ behind $\boldsymbol{E}_{\mathrm{m}}$. Thus the total delay between $\boldsymbol{E}_{\mathrm{m}}$ and the thermospheric density is around $4.5 \mathrm{~h}$. Therefore we can draw the conclusion that the mass density at altitudes around $400 \mathrm{~km}$ to $500 \mathrm{~km}$ responds to the change of solar wind input after $4.5 \mathrm{~h}$. Hedin (1981) concludes that the thermosphere responds 3 to $6 \mathrm{~h}$ after the energy deposition in the auroral ionosphere. Lathuillère et al. (2008) have studied how the disturbance coefficients of CHAMP and the model NRLMSISE-00 are related to the geomagnetic indices $a_{\mathrm{p}}$ and $a_{\mathrm{m}}$, a constant delay of $3 \mathrm{~h}$ is used for these indices. Moreover, Sutton et al. (2009) have investigated the delayed response of thermospheric mass density, which is also derived from the CHAMP measurements, to the Joule heating index, they also found that the response times of the thermosphere at equatorial regions are between 3.5 and $4 \mathrm{~h}$. They furthermore pointed out that the response times at northern mid latitudes are generally less than $2 \mathrm{~h}$, with lag times of $1 \mathrm{~h}$ or less observed closer to the pole. Their conclusions are quite consistent with the findings in Liu et al. (2010).

\subsection{The influence of different storm types}

The ICME-driven storms and CIR-driven storms are different in many aspects such as geomagnetic activities, ring current, aurora, convection, and so forth. Among the 31 storms used in this study, there are 24 ICME-driven storms and 8 CIRdriven storms. Half of the CIR-driven storms occurred in 2002. The strength of the 8 CIR-driven storms are weaker in terms of Dst index than the ICME-driven storms. The CIR-driven storms have a mean Dst value of $116 \mathrm{nT}$, while 

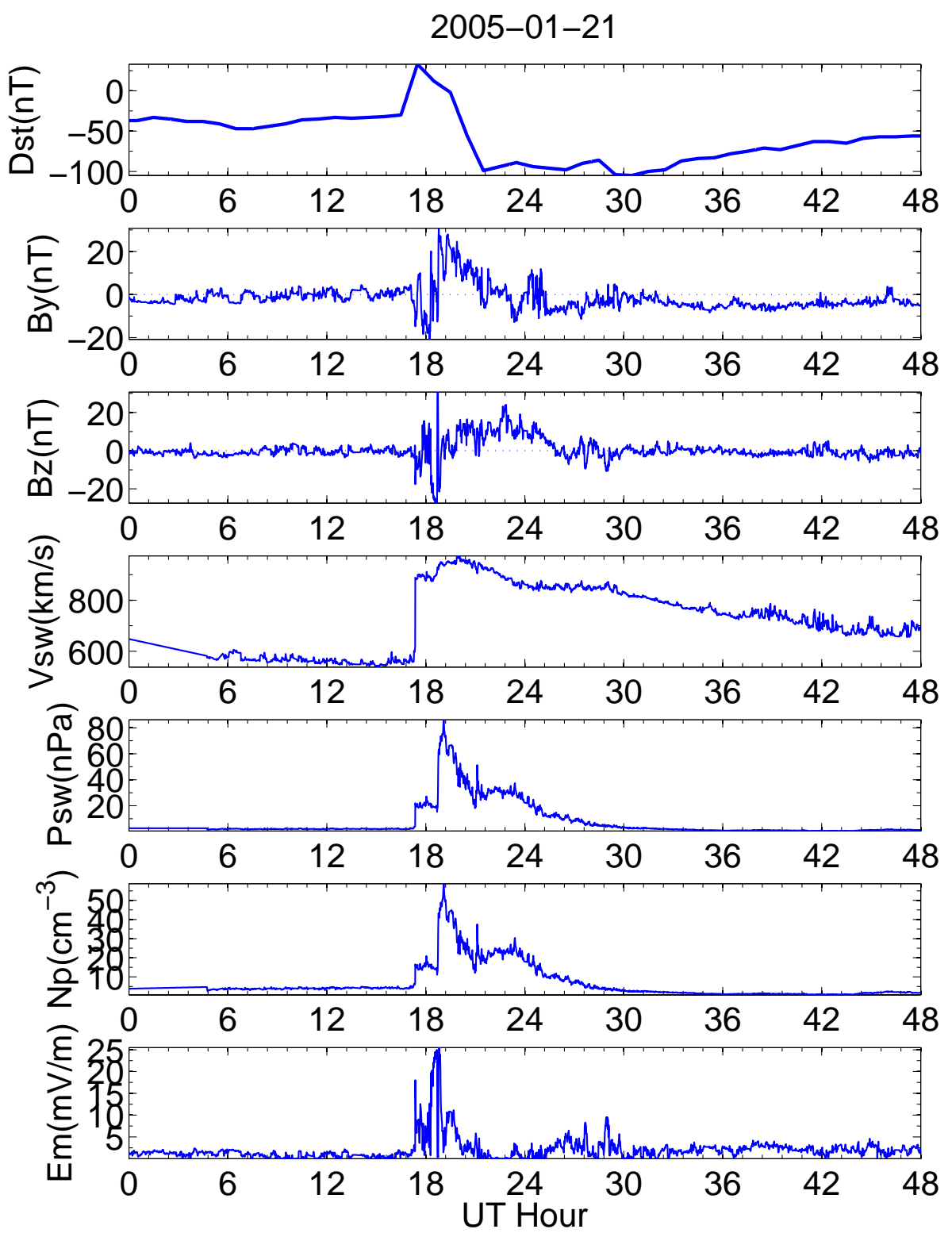

Fig. 9. The solar wind conditions for the storm during 21 to 22 January 2005 . From top to bottom are Dst index, IMF $B_{\mathrm{y}}$, IMF $B_{\mathrm{Z}}$, solar wind speed $v_{\mathrm{SW}}$, solar wind dynamic pressure $P_{\mathrm{SW}}$, solar wind proton number density $N_{\mathrm{p}}$ and merging electric field $\boldsymbol{E}_{\mathrm{m}}$.

the ICME-driven storms has a mean Dst value of $175 \mathrm{nT}$. In addition all the three super storms mentioned above belong to ICME-driven storms. On the contrary, the scale factor $a$ for the CIR-driven storms are interestingly, in general, larger than those for the ICME-driven storms, as is shown in Fig. 4. Borovsky and Denton (2006) have performed a systematic comparison between the properties of ICME-driven storms and CIR-driven storms. Among other properties the saturation of polar cap potential is investigated. They concluded that saturation of the polar cap occurs rarely for high-speeddriven storms, which belong to CIR-driven storms, but commonly for ICME-driven storms. Their conclusion gives us a good explanation why the CIR-driven storms have in com- mon higher $a$ values. If the polar cap potential doesn't saturate during a CIR-driven storm, the polar cap convection will be strengthened, and the Joule heating as well as the heating by the precipitating particles will be enhanced, which leads to enhancements of thermospheric densities. Therefore the merging electric field requires a larger $a$ for the density prediction. Denton et al. (2006) also concluded that the hot electron and hot ion temperatures in the plasma sheet are more and longer enhanced during CIR-driven storms than during ICME-driven storms. Recently, Lei et al. (2010b) have used CHAMP density data to study the thermospheric density response to solar wind high-speed streams/CIRs during the solar minimum of 2008. They concluded that thermospheric 
density observed by CHAMP is significantly disturbed due to CIRs even though their resultant recurrent geomagnetic activity is weak or moderate. This conclusion explains to some extent why the CIR-driven storms have larger $a$ values while the Dst index only attained moderate values compared to that during ICME-driven storms.

\subsection{The magnetic storm of 21 January 2005}

In Sect. 3 we noted already that the storm of 21 January 2005 is exceptional. This storm is a ICME-driven storm. The derived value of the scaling factor $a$ is outstanding in Fig. 4 both for CHAMP and GRACE. The value attained from correlation analysis between merging electric field and mass density variations is about a factor of 2 larger than the average value.

In an attempt to explain the exception we had a look at solar wind conditions during this storm. Figure 9 shows the temporal evolution of relevant quantities during 21 to 22 January 2005. The Dst evolution shows that the SSC begins at around 16:30 UT, then the main phase of the storm lasts about $4 \mathrm{~h}$ from 17:25 UT to 21:30 UT. Interestingly, after the IMF $B_{\mathrm{Z}}$ reaches its minimum $-27 \mathrm{nT}$ at around 18:30 UT, its value turns positive shortly before 19:00 UT and remains around $+10 \mathrm{nT}$ until $26 \mathrm{UT}$. This makes the storm very special, since during most part of the main phase $B_{\mathrm{Z}}$ is northward. This has also been reported by Du et al. (2008), but the mechanism of this storm event is still not clear. Du et al. (2008) attributed it to the storage of solar wind energy in the magnetotail and delayed release into the ring current.

Another outstanding feature is the extraordinary high dynamic pressure of the solar wind $\left(P_{\mathrm{sw}}\right)$, surpassing $85 \mathrm{nPa}$. This peak value is the highest record within the four years' observation. Furthermore, the pressure stayed high, above $30 \mathrm{nPa}$, for the subsequent $5 \mathrm{~h}$. The strong values of the pressure might be caused by an outstanding coronal mass ejection (McKenna-Lawlor et al., 2010). The merging electric field, shown in the bottom panel, is high only for a much shorter time. From the observation of this storm we suggest that the dynamic pressure may also have an influence on the storm-time density enhancement. In a future study we are planning to study the role of $P_{\mathrm{sw}}$ on the prediction of the thermospheric density.

\section{Conclusion}

In this study the model developed by Liu et al. (2010) has been utilized to predict the storm-time densities along both CHAMP and GRACE orbits. The properties of the model and the prediction results are compared at CHAMP and GRACE altitudes. The main conclusions of this study can be summarized as follows:

1. The orbit-averaged density along CHAMP and GRACE orbits both show a delayed response of $3 \mathrm{~h}$ to the solar wind inputs during the geomagnetic storms.
2. During the years 2002 to 2005 , the scale factor $a$ between the merging electric field and the thermospheric density for CHAMP observations remains in general at the same level. It fluctuates around the mean value, $a=0.5$, during the first 3 years, in 2005 it shows a slight increasing trend. The $a$ for GRACE densities exhibits a declining trend over the four years. These two different kinds of trends are caused by the combined effect of orbital decline and solar flux decay. The $a$ value at GRACE altitude is around $30 \%$ of that at CHAMP altitude.

3. The CIR-driven storms have commonly larger $a$ values than the ICME-driven storms.

4. The storm-time densities at CHAMP and GRACE altitudes show very similar distributions in shape, regardless of the orbital local time difference, but the amplitude for GRACE density is around $30 \%$ of that for CHAMP density.

Acknowledgements. We thank Eelco Doornbos for fruitful discussions about the CHAMP/GRACE density measurements and Matthias Förster for providing the ACE data. The operational support of the CHAMP mission by the German Aerospace Centre (DLR) and the financial support for the data processing by the Federal Ministry of Education and Research (BMBF), as part of the Geotechnology Programme, are gratefully acknowledged. One of the authors (Ruosi Liu) is supported by the German Academic Exchange Service (DAAD) and China Scholarship Council (CSC). This study is also supported by Doctoral Fund of Ministry of Education of China (Grant No. 200804860012) and the National Natural Science Foundation of China (Grant No. 40804049).

Topical Editor C. Jacobi thanks J. Lei and another anonymous referee for their help in evaluating this paper.

\section{References}

Borovsky, J. E. and Denton, M. H.: Differences between CMEdriven storms and CIR-driven storms, J. Geophys. Res., 111, A07S08, doi:10.1029/2005JA011447, 2006.

Bruinsma, S. L. and Forbes, J. M.: Global observation of traveling atmospheric disturbances (TADs) in the thermosphere, Geophys. Res. Lett., 34, L14103, doi:10.1029/2007GL030243, 2007.

Bruinsma, S., Tamagnan, D., and Biancale, R.: Atmospheric densities derived from CHAMP/STAR accelerometer observations, Planet. Space Sci., 52, 297-312, 2004.

Bruinsma, S., Forbes, J. M., Nerem, R. S., and Zhang, X.: Thermospheric density response to the 20-21 November 2003 solar and geomagnetic storm from CHAMP and GRACE accelerometer data. J. Geophys. Res., 111, A06303, doi:10.1029/2005JA011284, 2006.

Burke, W. J., Huang C. Y., Marcos, F. A., and Wise, J. O.: Interplanetary control of thermospheric densities during large magnetic storms, J. Atmos. Solar-Terr. Phys., 69, 279-287, 2007.

Burke, W. J., Lin, C. S., Hagan, M. P., Huang, C. Y., Weimer, D. R., Wise, J. O., Gentile, L. C., and Marcos, F. A.: Storm time global thermosphere: A driven-dissipative thermodynamic system, J. Geophys. Res., 114, A06306, doi:10.1029/2008JA013848, 2009. 
Crowley, G.: Dynamics of the Earth's thermosphere: A review, Rev. Geophys., 29, 1143-1165, 1991.

Denton, M. H., Borovsky, J. E., Skoug, R. M., Thomsen, M. F., Lavraud, B., Henderson, M. G., McPherron, R. L., Zhang, J. C., and Liemohn, M. W.: Geomagnetic storms driven by ICMEand CIR-dominated solar wind, J. Geophys. Res., 111, A07S07, doi:10.1029/2005JA011436, 2006.

Doornbos, E., IJssel, J., Lühr, H., Förster, M., and Koppenwallner, G.: Neutral density and crosswind determination from arbitrarily oriented multiaxis accelerometers on satellites, J. Spacraft Rockets, 47(4), 580-589, doi:10.2514/1.48114, 2010.

Du, A. M., Tsurutani, B. T., and Sun, W.: Anomalous geomagnetic storm of 21-22 January 2005: A storm main phase during northward IMFs, J. Geophys. Res., 113, A10214, doi:10.1029/2008JA013284, 2008

Forbes, J. M., Gonzalez, R., Marcos, F. A., Revelle, D., and Parish, H.: Magnetic storm response of lower thermosphere density, J. Geophys. Res., 101(A2), 2313-2319, doi:10.1029/95JA02721, 1996.

Forbes, J. M., Lu, G., Bruinsma, S., Nerem, R. S., and Zhang, X.: Thermosphere density variations due to the 15-24 April 2002 solar events from CHAMP/STAR accelerometer measurements, J. Geophys. Res., 110, A12S27, doi:10.1029/2004JA010856, 2005.

Fuller-Rowell, T. J., Codrescu M. V., Moffett, R. J., and Quegan, S.: Response of the thermosphere and ionosphere to geomagnetic storms, J. Geophys. Res., 99(A3), 3893-3914, 1994.

Hedin, A. E., Spencer, N. W., Mayr, H. G., and Porter, H. S.: Semi-empirical modeling of thermospheric magnetic storms, J. Geophys. Res.,86, 3515-3518, doi:10.1029/JA086iA05p03515, 1981.

Hedin, A. E.: Extension of the MSIS thermosphere model into the middle and lower atmosphere, J. Geophys. Res., 96(A2), 11591172, 1991.

Lathuillère, C., Menvielle, M., Marchaudon, A., and Bruinsma, S.: A statistical study of the observed and modeled global thermosphere response to magnetic activity at middle and low latitudes, J. Geophys Res., 113, A07311, doi:10.1029/2007JA012991, 2008.

Lei, J., Thayer, J. P., Burns, A. G., Lu, G., and Deng, Y.: Wind and temperature effects on thermosphere mass density response to the November 2004 geomagnetic storm, J. Geophys. Res., 115, A05303, doi:10.1029/2009JA014754, 2010a.

Lei, J., Thayer, J. P., Wang, W., and McPherron, R. L.: Impact of CIR storms on thermosphere density variability during the solar minimum of 2008, Solar Phys., in press, doi:10.1007/s11207010-9563-y, 2010b.

Liu, H. and Lühr, H.: Strong disturbance of the upper thermospheric density due to magnetic storms: CHAMP observations, J. Geophys. Res., 110, A09S29, doi:10.1029/2004JA010908, 2005.

Liu, L., Wan, W., Ning, B., Pirog, O. M., and Kurkin, V. I.: Solar activity variation of the ionospheric peak electron density, J. Geophys. Res., 111, A08304, doi:10.1029/2006JA011598, 2006.

Liu, R., Lühr, H., Doornbos, E., and Ma, S.-Y.: Thermospheric mass density variations during geomagnetic storms and a prediction model based on the merging electric field, Ann. Geophys., 28, 1633-1645, doi:10.5194/angeo-28-1633-2010, 2010.
Matuura, N.: Theoretical models of ionospheric storms, Space Sci. Rev., 13, 124-189, 1972.

McKenna-Lawlor, S., Li, L., Dandouras, I., Brandt, P. C., Zheng, Y., Barabash, S., Bucik, R., Kudela, K., Balaz, J., and Strharsky, I.: Moderate geomagnetic storm (21-22 January 2005) triggered by an outstanding coronal mass ejection viewed via energetic neutral atoms, J. Geophys. Res., 115, A08213, doi:10.1029/2009JA014663, 2010.

Müller, S., Lühr, H., and Rentz, S.: Solar and magnetospheric forcing of the low latitude thermospheric mass density as observed by CHAMP, Ann. Geophys., 27, 2087-2099, doi:10.5194/angeo27-2087-2009, 2009.

Picone, J. M., Hedin, A. E., Drob, D. P., and Aikin, A. C.: NRLMSISE-00 empirical model of the atmosphere: statistical comparisons and scientific issues, J. Geophys. Res., 107(A12), 1468-1483, doi:10.1029/2002JA009430, 2002.

Prölss, G. W.: Magnetic storm associated perturbations of the upper atmosphere: Recent results obtained by satellite-borne gas analyzers, Rev. Geophys., 18, 183-202, doi:10.1029/RG018i001p00183, 1980.

Rentz, S. and Lühr, H.: Climatology of the cusp-related thermospheric mass density anomaly, as derived from CHAMP observations, Ann. Geophys., 26, 2807-2823, doi:10.5194/angeo-262807-2008, 2008.

Richards, P. G., Fennelly, J. A., and Torr, D. G.: EUVAC: A solar EUV flux model for aeronomic calculations, J. Geophys. Res., 99, 8981-8992, 1994.

Romanoysky, Y. A. and Katyushina, V. V.: On thermospheric composition and temperature variations during geomagnetic disturbances, Space Res., 14, 163-167, 1974.

Sutton, S. K., Forbes, R. S., and Nerem, R. S.: Global thermospheric neutral density and wind response to the severe 2003 geomagnetic storms from CHAMP accelerometer data, J. Geophys. Res., 110, A09S40, doi:10.1029/2004JA010985, 2005.

Sutton, E. K., Forbes, J. M., and Knipp, D. J.: Rapid response of the thermosphere to variations in Joule heating, J. Geophys. Res., 114, A04319, doi:10.1029/2008JA013667, 2009.

Weimer, D. R., Ober, D. M., Maynard, N. C., Collier, M. R., McCormas, D. J., Ness, N. F., Smith, C. W., and Watermann, J.: Predicting interplanetary magnetic field (IMF) propagation delay times using the minimum variance technique, J. Geophys. Res., 108(A1), 1026, doi:10.1029/2002JA009405, 2003.

Wilson, G. R., Weimer, D. R., Wise, J. O., and Marcos, F. A.: Response of the thermosphere to Joule heating and particle precipitation, J. Geophys. Res., 111, A10314, doi:10.1029/2005JA011274, 2006.

Zhou, Y. L., Ma, S. Y., Lühr, H., Xiong, C., and Reigber, C.: An empirical relation to correct storm-time thermospheric mass density modeled by NRLMSISE-00 with CHAMP satellite air drag data, Adv. Space Res., 43, 819-828, 2009. 